\title{
Correlações entre Assertividade, Condições Laborais, Estresse e Depressão de Servidores do INSS/RO
}

\author{
Leandro Aparecido Fonseca Missiatto ${ }^{1}$ \\ ${ }^{1}$ Faculdade de Ciências Biomédicas de Cacoal, RO, Brasil. \\ Tomás Daniel Menéndez Rodríguez ${ }^{2}$ \\ ${ }^{2}$ Universidade Federal de Rondônia, RO, Brasil. \\ Fabio Biasotto Feitosa ${ }^{2}$ \\ ${ }^{2}$ Universidade Federal de Rondônia, RO, Brasil.
}

Resumo: Este artigo é resultado de um estudo que teve como objetivo analisar as correlações entre assertividade, condições laborais, estresse percebido e depressão de servidores públicos do Instituto Nacional de Seguridade Social (INSS) em Rondônia. Fizeram parte deste estudo 132 servidores efetivos do INSS de Rondônia, distribuídos pela capital e interior do Estado (61,4\% da capital; 31,6\% do interior). Foram utilizados como instrumentos: Questionário de Condições de trabalho, Escala de Estresse Percebido (PSS-14), Patient Health Questionnaire-9(PHQ-9) e Escala Fatorial de Extroversão (EFEx). Os dados foram tratados por estatísticas descritiva e inferencial utilizando-se o sistema SPSS 2.0. Os resultados não identificaram correlação significativa entre assertividade com estresse percebido e depressão, todavia a relação entre depressão, estresse percebido com as condições laborais foram relevantes. Servidores com baixa autonomia no trabalho, dificuldades em negociar necessidades com a chefia, dificuldades em compreender as deliberações superiores e que não sabiam as expectativas sobre seu trabalho relataram mais sintomas depressivos e estresse. Os resultados sugerem que a depressão e em parte o estresse percebido estão associados às condições subjetivas de trabalho e não à assertividade no público estudado. Tais informações podem contribuir para implementação de políticas de saúde específicas para este público.

Palavras-chave: Saúde mental. Assertividade. Estresse. Condições de trabalho. Servidores públicos.

\section{Correlations between Assertiveness, Labor Conditions, Stress and Depression of Government Employees INSS/RO}

\begin{abstract}
This article is the result of a study that aimed to analyze the correlations between assertiveness, labor conditions, perceived stress and depression of government employees of the National Social Security Institute (INSS) in Rondônia. This study included 132 effective employees of INSS of Rondônia, distributed by the state capital and the interior of the state $(61.4 \%$ of the capital, $31.6 \%$ of the interior). The following instruments were used: Work Conditions Questionnaire, Perceived Stress Scale (PSS-14), Patient Health Questionnaire-9 (PHQ-9) and Factorial Extroversion Scale (EFEx). Data were treated by descriptive and inferential statistics using the SPSS 2.0 system. The results did not identify significant correlation between assertiveness with perceived stress and depression; however, the relationship between depression, perceived stress and labor conditions was relevant. Employees with low autonomy at work, difficulties in negotiating needs with the boss, difficulties in understanding the superior deliberations and who did not know the expectations about their work reported more depressive symptoms and stress. The results suggest that depression and in part perceived stress are associated with subjective work conditions rather than with assertiveness for the employees studied. Such information may contribute to the implementation of specific health policies for these employees.
\end{abstract}

Keywords: Mental health. Assertiveness. Stress. Labor conditions. Government employees. 


\title{
Correlaciones entre Asertividad, Condiciones Laborales, Estrés y Depresión de Servidores del INSS/RO
}

\begin{abstract}
Resumen: Este artículo es el resultado de un estudio que tuvo como objetivo analizar las correlaciones entre asertividad, condiciones laborales, estrés percibido y depresión de servidores públicos del Instituto Nacional de Seguridad Social (INSS) en Rondônia. Este estudio incluyó a 132 servidores efectivos del INSS de Rondônia, distribuidos por la capital e interior del Estado (61,4\% de la capital, 31,6\% del interior). Se utilizaron los siguientes instrumentos: Cuestionario de Condiciones de trabajo, Escala de Estrés Percibido (PSS-14), Patient Health Questionnaire-9 (PHQ-9) y Escala Factorial de Extroversión (EFEx). Los datos se trataron mediante estadística descriptiva e inferencial utilizando el sistema SPSS 2.0. Los resultados no identificaron correlación significativa entre asertividad con estrés percibido y depresión, sin embargo, la relación entre la depresión, el estrés percibido y las condiciones laborales fueron relevantes. Los servidores con baja autonomía en el trabajo, dificultades en negociar necesidades con sus jefes, dificultades para comprender las deliberaciones superiores y no conocer las expectativas sobre su trabajo informaron más síntomas depresivos y estrés. Los resultados sugieren que la depresión y en parte, el estrés percibido, están asociados a las condiciones subjetivas de trabajo y no con asertividad en el público estudiado. Esta información puede
\end{abstract}

Palabras clave: Salud mental. Asertividad. Estrés. Condiciones de trabajo. Servidores públicos.

\section{Introdução}

Este artigo resulta de um estudo que objetivou verificar a interação estabelecida entre depressão, estresse psicológico, assertividade e as condições de trabalho de funcionários públicos do Instituto Nacional de Seguridade Social de Rondônia (INSS/RO). A ideia inicial foi de que a assertividade funcionaria como proteção à saúde mental de servidores do Instituto Nacional de Seguridade Social (INSS) do Estado de Rondônia, no tocante ao estresse e depressão, e as condições negativas de trabalho como agravantes a estes transtornos.

O interesse pela saúde mental de funcionários públicos se deve à importância da atividade pública para o funcionamento social e manutenção do Estado Democrático. Neste sentido, uma das instituições de singular relevância para a performance do Estado é o Instituto Nacional de Seguridade Social (INSS), tendo em vista a amplidão de serviços que oferece e o perfil da clientela que atende.

O INSS é uma instituição criada pelo então presidente da república Fernando Collor de Melo, em 27 de junho de 1990. Trata-se de uma autarquia do governo federal atualmente vinculada ao Ministério do Desenvolvimento Social. Uma de suas funções é garantir a seguridade social aos brasileiros, destacando o pagamento das aposentadorias, pensões e auxílios.
A mais importante função do INSS é a operacionalização dos direitos da população que está inserida no Regime Geral da Previdência Social (RGPS), que atualmente atinge a marca de pouco mais de 54 milhões de contribuintes (Brasil, 2017). Conforme a Constituição Federal de 1988, o RGPS possui caráter de filiação obrigatória e deve respeitar as estratégias de seguridade social, implementadas pelos ministérios (Constituição da República Federativa do Brasil, 1988).

Embora sejam de grande importância as atividades desenvolvidas pelos servidores do Instituto Nacional de Seguridade Social, pouco se sabe sobre estes servidores. Questões como sua saúde, qualidade de vida e outras variáveis, elementos significativos para qualidade da prestação de serviços aos usuários, são ainda pouco estudadas (Galvão, 2015). A rarefeita quantidade de estudos nesta área não possibilita compreender quais são as variáveis de risco à saúde dos trabalhadores do INSS, bem como dificulta na elaboração de políticas institucionais que promovam saúde e bem-estar destes trabalhadores.

Desta forma, a pesquisa apresentada é o resultado de um estudo que teve como objetivo verificar as inter-relações estabelecidas entre as seguintes variáveis: depressão, estresse psicológico, condições de trabalho e assertividade. A hipótese principal era de 
que a assertividade se relacionaria de forma significativa com menores níveis de depressão e estresse o que poderia inferir uma ação protetiva a estes transtornos, enquanto as condições negativas de trabalho atuariam de forma inversa.

\section{Interações entre depressão, estresse, assertividade e condições de trabalho na literatura}

A depressão é um transtorno de humor que vem acometendo um número crescente de pessoas pelo mundo (Motta, 2011) e no Brasil é uma importante causa de afastamento do trabalho (Cavalheiro \& Tolfo, 2011; Cavalheiro, 2010). Os desdobramentos das alterações provocadas por este tipo de doença repercutem na economia do país, na construção de estratégias de saúde coletiva e no bem-estar das pessoas (Feitosa, 2014). Estudos (Enns, Currie, \& Wang, 2015; Santos et al., 2011; Uliaszek et al., 2010; Flynn \& Rudolph, 2011) têm demonstrado a correlação desse transtorno com a assertividade, condições de trabalho e o estresse.

Segundo Lazarus e Folkman (1984, p. 19), estresse é "uma relação particular entre a pessoa e o ambiente, avaliada pelo sujeito como desestabilizadora ou superior aos seus recursos disponíveis, e colocando em risco seu bem-estar". A principal ideia deste modelo explicativo do estresse consiste no fato de que situações podem ser estressantes para algumas pessoas e não para outras, o que determina isso é a percepção do sujeito sobre os eventos possivelmente estressores.

No contexto interpessoal o estresse tem-se mostrado significativo para o surgimento da depressão. Conforme Feitosa (2014), o estresse interpessoal, aquele ocorrido em razão das relações e demandas interpessoais como iniciar uma conversação, resolver um conflito e defender direitos, é importante elemento para origem da depressão, de forma que saber interagir adequadamente com as pessoas é crucial para a saúde mental. Neste sentido, Uliaszek et al. (2010) realizaram estudo com 603 estudantes de escolas secundárias das cidades de Chicago e Los Angeles, tendo como fatores de risco distúrbios emocionais. A pesquisa identificou correlação em eventos interpessoais estressantes com depressão $(r=0,32 ; p<0,001)$, maior do que eventos estressantes sem cunho interpessoal $(r=0,25 ; p<0,001)$. Portanto, o estudo conclui que a qualidade das relações interpessoais é indicador para o possível surgimento e desenvolvimento do estresse e depressão.
No contexto do trabalho, o estresse também manifesta correlação significativa com a depressão. $\mathrm{O}$ estudo de Gherardi-Donato, Cardoso, Teixeira, Pereira e Resdorfer (2015), com 310 técnicos de enfermagem de um hospital-escola do estado de São Paulo, observou a associação entre alto nível de estresse laboral, depressão e afastamento no trabalho $(\mathrm{p}<0,001)$. Cerca de $50 \%$ afastaram-se do trabalho por motivo de doença no último ano e a maioria dos entrevistados referiu ter ao menos uma doença crônica $(76,1 \%)$. Segundo os dados da pesquisa, técnicos de enfermagem com alto nível de estresse laboral apresentavam prevalência de depressão 2,69 vezes maior em comparação àqueles sem estresse laboral. Os resultados fortalecem a ideia de que o estresse laboral é importante para o desenvolvimento de depressão, e que o controle do estresse no local de trabalho é fundamental para prevenção de sintomas depressivos no trabalhador.

A natureza do estresse laboral em grande parte se deve às condições de trabalho a que são submetidos diariamente os trabalhadores. Quando falamos em condições de trabalho estamos nos referindo ao conjunto de circunstâncias em que as atividades laborais são desenvolvidas, bem como sua capacidade de repercutir significativamente na experiência laborativa do trabalhador, tanto quanto em suas relações de trabalho (Blanch, 2003). Deste modo, as condições de trabalho abarcam elementos que vão desde aspectos mais elementares como ambiente físico, jornada de trabalho, a elementos mais complexos como relacionamento interpessoal com colegas e chefia, bem como autonomia no trabalho.

Interessado nas condições de trabalho e sua interferência direta com o estresse de médicos, Santos et al. (2011) realizaram um estudo com 321 destes profissionais de saúde do município de João Pessoa (PB). Os resultados mostraram que condições de trabalho - como relacionamento com a equipe $(0,881)$, com os colegas médicos $(0,865)$, com a chefia $(0,806)$, restrição da autonomia profissional $(0,796)$ e trabalho noturno $(0,808)$ - são elementos do trabalho que estão envolvidos no desenvolvimento do estresse dos médicos pesquisados. Os resultados apresentaram consistência e significância pelo modelo Alfa de Cronbach e análise fatorial. Este estudo demonstra correlação significativa $(p<0,05)$ entre as condições de trabalho e o desenvolvimento do estresse em trabalhadores.

No Canadá, pesquisadores (Enns et al., 2015) realizaram estudo com uma amostra de 17.437 enfermei- 
ros, utilizando de análise transversal e secundária dos dados, para delinearem a influência da autonomia no trabalho como fator de risco para depressão de enfermeiros. Os resultados demonstraram que a depressão é significativamente maior no grupo de enfermeiros que tem menor autonomia laboral $(\mathrm{p}<0,001)$, o que sugere o impacto que esta condição de trabalho exerce na saúde mental destes profissionais, além de indicar que políticas de expansão da autonomia no contexto de trabalho dos enfermeiros pode contribuir significativamente para o enfrentamento da depressão nestes profissionais de saúde.

Nas condições interpessoais de trabalho, a assertividade tem demonstrado significativa relação com a etiologia da depressão. Segundo Bolsoni-Silva e Carrara (2010), a assertividade pode ser definida como:

o processo pelo qual o indivíduo (emissor) expressaria, de forma adequada, sentimentos e pensamentos, ou seja, utilizando entonação, latência e fluência de falas apropriadas, ouvindo o receptor para, então, responder, de forma a atingir seus objetivos sem prejudicar as relações futuras (p. 333).

Sendo assim, pessoas assertivas ao expressarem adequadamente sentimentos e pensamentos, tenderiam a diminuir a probabilidade do surgimento do estresse interpessoal e minimizariam o seu impacto na saúde. Em uma pesquisa realizada por Razayat e Neyeri (2014), na Universidade do Teerã com 250 estudantes de enfermagem, cujo objetivo foi investigar a relação estabelecida entre assertividade e níveis de depressão, identificou-se que $55,6 \%$ dos alunos apresentaram níveis médios de assertividade e $38,7 \%$ dos participantes estavam sofrendo de depressão leve a grave. $\mathrm{O}$ teste de correlação apresentou relação inversa significativa $(r=-0,314, p<0,001)$ entre assertividade e depressão. Ou seja, os dados apontam a assertividade como fator de proteção contra a depressão. Neste estudo foi detectado que $72,0 \%$ dos estudantes que tinham interesse no seu campo de estudo não apresentaram sintomas de depressão. A hipótese é de que a satisfação com o estudo funcione como força motriz para assertividade e redução da prevalência de depressão. Desta forma, estar satisfeito com aquilo que se faz pode de alguma forma se associar com a expressão da assertividade e por consequência com a expressão da depressão.

Contudo, estudo conduzido por Eslami, Rabiei, Afzali, Hamidizadeh e Masoudi (2016), com 126 estu- dantes do Irã, cujo objetivo foi determinar a eficácia do treinamento de assertividade nos níveis de estresse, ansiedade e depressão dos estudantes do ensino médio, constatou ser significativo o treinamento de assertividade para controle de estresse e ansiedade, todavia não houve diferença significativa para depressão $(\mathrm{p}<0,05)$. O estudo aponta as seguintes explicações para estes resultados. O primeiro estaria no fato de a depressão ser mais complexa que a ansiedade e o estresse, estando profundamente arraigada nos estudantes e, portanto, sendo de mais demorada solução. A segunda estaria no fato de que o estresse e a ansiedade geralmente antevêm à depressão, desta forma, a eficácia do treinamento de assertividade talvez se associe melhor à prevenção que para tratamento.

Os dados das várias pesquisas aqui apresentadas retratam a frequente relação existente entre estresse, depressão, assertividade e condições de trabalho. Com base nestes elementos, o objetivo deste estudo foi analisar a inter-relação estabelecida entre depressão, estresse, condições de trabalho e assertividade de servidores públicos do INSS em Rondônia.

\section{Método}

\section{Participantes}

Fizeram parte deste estudo 132 servidores efetivos do INSS de Rondônia, distribuídos pela capital e interior do Estado (61,4\% da capital; 31,6\% do interior). Atualmente o INSS de Rondônia conta com 327 servidores, destes, 169 estão lotados na capital e 158 no interior. Deste modo, a quantidade de sujeitos participantes compreendeu $40 \%$ do total de servidores ativos do INSS/RO.

Os participantes concordaram em participar do estudo por meio do Termo de Consentimento Livre e Esclarecido. Foram 39 pessoas do sexo masculino e 93 do sexo feminino, entre idades de 18 a 65 anos (mediana $=53$; média $=47,5$; desvio-padrão $=13,31$ ). Dos pesquisados $38,6 \%$ possuíam ensino superior e $61,4 \%$ ensino médio. Permaneciam estudando um total de $35,6 \%$. A maior parte dos servidores ocupavam cargo de nível médio (82,6\%), apenas $2,3 \%$ eram ocupantes de cargo de nível superior e 12,9\% exerciam a função de gestor.

\section{Material}

Foram usados os seguintes instrumentos para coleta de dados de condições de trabalho e avaliação 
do estresse, depressão e assertividade, aplicados respectivamente nesta sequência.

Questionário sobre condições de trabalho. Este instrumento, desenvolvido pelos pesquisadores, objetivou coletar dados referentes às condições de trabalho dos servidores do INSS. Consistiu em nove perguntas do tipo "sim" e "não", autoaplicável, que verificou as percepções dos servidores quanto as suas condições de trabalho (Anexo 1). O questionário compreendia perguntas quanto a autonomia para gerenciar o trabalho $(1,2,6)$, apoio recebido por parte da chefia e dos colegas $(3,7,8,9)$, clareza nas exigências $e$ definição das funções $(4,5)$.

Escala de Estresse Percebido (PSS-14). Esta escala foi elaborada por Cohen, Karmack e Mermelsteinm (1983). Trata-se de um instrumento autoaplicável de avaliação mundialmente utilizado em pesquisas do estresse psicológico. É composta por quatorze itens que acessam a frequência com que no último mês o indivíduo lidou com situações inesperadas, incontroláveis ou que representam uma sobrecarga, exigindo que avalie, por exemplo, a sua percepção de controle e o seu estado emocional diante desses eventos estressantes (Cohen \& Williamson, 1988). Existem sete itens positivos $(4,5,6,7,9,10$ e 13) que precisam ser revertidos da seguinte maneira $(0=4,1=3,2=2,3=1,4=0)$. Após a reversão desses sete itens, os escores dos quatorze itens são somados, obtendo-se o escore bruto que representa o nível de estresse psicológico. A versão original da escala obteve índices aceitáveis de precisão e validade de critério (Feitosa, Silva, \& Bezerra, 2015), o reteste apresentou estabilidade temporal $(r=0,79$, $\mathrm{p}<0,001$ ) e quanto à consistência interna os resultados são positivos (Alpha $=0,85$ no teste e Alpha $=0,86$ no reteste). $\mathrm{O}$ estudo indica ainda que os escores médios da amostra da PSS-14 teve distribuição normal, livres do efeito "chão" e do efeito "teto". Este instrumento se adequou aos objetivos da pesquisa por destacar a sintomatologia psicológica do estresse. Para atribuição de significativos níveis de estresse, considerou-se o limiar de 27,3 pontos na PSS-14, nível preditivo para o fenômeno, conforme o estudo de Feitosa et al. (2015).

Patient Health Questionnaire-9 (PHQ-9). Conforme Santos et al. (2013), o PHQ-9 é um instrumento de aplicação relativamente rápida. O questionário é composto por nove questões, o que seria uma vantagem em estudos epidemiológicos, em comparação a outros atualmente validados para o Brasil, que se caracterizam por sua extensão. As nove questões verificam os sintomas descritos para depressão maior no Manual Diagnóstico e Estatístico dos Transtornos Mentais (DSM-V). O instrumento tem a vantagem de ser autoaplicado, utilizando-se de uma escala Likert que avalia a frequência dos sintomas nas últimas duas semanas. Em estudo de Santos et al. (2013), com intuito de verificar a validade do PHQ-9 em 447 participantes (191 homens e 256 mulheres), verificou-se como de máxima sensibilidade $(77,5 \%$; $61,5-89,2)$ e especificidade $(86,7 \%$; 83,0-89,9). Dessa forma, o PHQ-9 mostrou-se adequado para rastreamento de episódio depressivo maior. Os níveis de depressão podem ser classificados de acordo com as seguintes faixas de pontuação: minimamente deprimido (0-4), pouco deprimido (5-9), moderadamente deprimido (10-14), muito deprimido (15-19) e severamente deprimido (20-27). No presente trabalho, optou-se por atribuir a categoria não deprimido aos participantes que pontuaram zero.

Escala Fatorial de Extroversão (EFEx). Trata-se de uma escala objetiva de autorrelato, composta por 57 itens onde estão descritos sentimentos, crenças e atitudes associados a traços de personalidade importantes para a avaliação de Extroversão. Uma das facetas do instrumento é a averiguação da assertividade, sua terceira dimensão. A assertividade é avaliada por subitens que descrevem características como assertividade, liderança, nível de atividade e motivação. Pessoas com altos escores na faceta de assertividade tendem a ter "facilidade para tomar decisões, desenvoltura para expressar e defender suas opiniões, tendem a ser muito ativas e relatam não ter receio em envolver-se em muitas atividades" (Nunes, 2007; p. 103). Conforme o manual da EFEx, foi realizada pesquisa que objetivou verificar a validade da EFEx. Participaram deste estudo 1.084 indivíduos de ambos os sexos e de vários estados brasileiros. A dimensão da assertividade (E3) obteve boa consistência interna no instrumento calculado por Alpha de Cronbach $(0,78)$. Considerando a qualidade do instrumento para verificação da assertividade, utilizou-se então, a dimensão E3, responsável pela avaliação da assertividade nos servidores do INSS/RO.

\section{Procedimentos}

A proposta de estudo foi apresentada à gerência geral do INSS de Rondônia que apoiou a pesquisa e concedeu que fosse desenvolvida nas cidades que possuíam suas agências. O estudo foi submetido à avaliação do Comitê de Ética em Pesquisa, sendo aprovado sob o número CAAE: 55655916.0.0000.5300. AA aplica- 
ção dos instrumentos aconteceu de forma individual e coletiva, respeitando a disponibilidade de local de cada agência do INSS. Os participantes receberam orientações sobre a pesquisa, e em posse do Termo de Consentimento Livre e Esclarecido, puderam optar pela participação no estudo. Os instrumentos foram autoaplicados, com auxílio de orientações do pesquisador.

\section{Análise de Dados}

A análise dos dados foi realizada de acordo com os critérios dos instrumentos aplicados e tratados por estatísticas descritiva e inferencial, utilizando-se o pacote SPSS para Windows versão 20.0. Considerando o tamanho da amostra, e a característica dos testes, utilizou-se para análise de dados o teste não-paramétrico de Mann-Whitney, adequados para amostras pequenas e sem distribuição homogêneas, garantido análises de significância e correlação de grupos independentes mais precisos (Hackbarth \& Stein, 2003).

\section{Resultados}

Os resultados mostram haver uma relação inversa entre a assertividade, verificada pela dimensão E3 da Escala Fatorial de Extroversão, e estresse e depressão, verificados respectivamente pelos PSS-14 e PHQ-9, mas não fortes o suficiente para indicar significância (Tabela 1), o que significa dizer que a assertividade é menor em pessoas deprimidas e estressadas do que naquelas que não manifestam estes transtornos, todavia não podemos falar que seja significativa esta relação para os dois grupos.

Tabela 1

Análise da correlação da assertividade com o estresse percebido e a depressão $(\mathrm{N}=132)$.

\begin{tabular}{lcc}
\hline \multicolumn{3}{l}{ Escala Fatorial de Extroversão - E3 } \\
\hline MHgnitude & Significância \\
\hline PHQ-9 & $-0,095$ & 0,280 \\
PSS-14 & $-0,046$ & 0,597 \\
\hline
\end{tabular}

O Questionário de condições de trabalho identificou que as condições subjetivas de trabalho, como atividades desenvolvidas em equipe, saber o que esperam de seu trabalho, negociação com o chefe, autonomia e as características das deliberações superiores se relacionam de forma significativa com o estresse percebido e os sintomas de depressão. Estas informações permitem afirmar que para os servidores do INSS/RO certas condições de trabalho importam para proteger ou desenvolver/agravar o estresse e depressão de seus servidores.

A condição de trabalho descrita no estudo como autonomia para gerenciar o trabalho (Tabela 2), possui relação inversa com a depressão já que a média daqueles que perceberam não possuir autonomia para gerenciar o trabalho foi quase duas vezes maior em relação àqueles que compreendiam possuir tal autonomia. $\mathrm{O}$ estudo identificou também que a falta de autonomia para gerenciar o trabalho impactou mais para o desenvolvimento ou percepção de sintomas depressivos $(\mathrm{p}<0,001)$ do que a ausência de autonomia para gerir direitos pessoais como folgas e férias ( $p=0,538$ ) ou coordenar equipe $(p=0,838)$.

\section{Tabela 2}

Análise da relação entre a percepção de autonomia dos servidores do INSS de Rondônia e sintomas de estresse percebido e depressão.

\begin{tabular}{|c|c|c|c|c|}
\hline \multicolumn{5}{|c|}{ Autonomia para gerenciar o trabalho } \\
\hline & & & PHQ-9 & PSS-14 \\
\hline \multirow{7}{*}{$\begin{array}{l}\text { Autonomia } \\
\text { para } \\
\text { gerenciar o } \\
\text { trabalho }\end{array}$} & \multirow{3}{*}{ Sim } & $\mathrm{N}$ & 60,000 & 60,000 \\
\hline & & Média & 6,630 & 25,920 \\
\hline & & D.P. & 5,500 & 9,080 \\
\hline & \multirow{4}{*}{ Não } & $\mathrm{N}$ & 72,000 & 72,000 \\
\hline & & Média & 11,210 & 28,320 \\
\hline & & D.P. & 6,000 & 7,050 \\
\hline & & Sig. & $0,000^{*}$ & 0,094 \\
\hline \multirow{7}{*}{$\begin{array}{l}\text { Coordena } \\
\text { equipe }\end{array}$} & \multirow{3}{*}{ Sim } & $\mathrm{N}$ & 36,000 & 36,000 \\
\hline & & Média & 8,870 & 25,780 \\
\hline & & D.P. & 5,460 & 10,310 \\
\hline & \multirow{4}{*}{ Não } & $\mathrm{N}$ & 96,000 & 96,000 \\
\hline & & Média & 9,190 & 27,770 \\
\hline & & D.P. & 6,470 & 7,080 \\
\hline & & Sig. & 0,838 & 0,252 \\
\hline \multirow{7}{*}{$\begin{array}{l}\text { Autonomia } \\
\text { para } \\
\text { organizar } \\
\text { o trabalho, } \\
\text { folgas e férias }\end{array}$} & \multirow{3}{*}{ Sim } & $\mathrm{N}$ & 102,000 & 102,000 \\
\hline & & Média & 8,880 & 27,420 \\
\hline & & D.P. & 6,090 & 7,930 \\
\hline & \multirow{4}{*}{ Não } & $\mathrm{N}$ & 30,000 & 30,000 \\
\hline & & Média & 9,970 & 26,570 \\
\hline & & D.P. & 6,580 & 8,740 \\
\hline & & Sig. & 0,538 & 0,565 \\
\hline
\end{tabular}

${ }^{*} \mathrm{p}<0,001$.

Em relação à condição de trabalho apoio por parte da chefia e dos colegas (Tabela 3), nota-se que aqueles servidores que não se sentiam confortáveis em negociar 
suas necessidades com seus chefes (sejam de trabalho ou pessoais), asseveraram ser moderadamente deprimidos, enquanto que os servidores que se sentiam bem em negociações com suas chefias indicaram níveis de menor depressão $(\mathrm{p}<0,001)$. Outro dado interessante quanto às relações interpessoais é que os servidores que trabalhavam em equipe manifestaram estresse, enquanto aqueles que desenvolviam suas atividades individualmente não se perceberam estressados $(p<0,05)$ e que o primeiro grupo manifestou mais sintomas depressivos que o segundo $(\mathrm{p}<0,01)$. Não houve diferenças significativas entre receber ou não apoio do chefe em iniciativas, e entre receber ou não ajuda dos colegas no trabalho.

\section{Tabela 3}

Análise da relação entre percepção de apoio dos servidores do INSS de Rondônia e sintomas de estresse percebido e depressão.

\begin{tabular}{|c|c|c|c|c|}
\hline \multicolumn{5}{|c|}{ Apoio recebido por parte da chefia e dos colegas } \\
\hline & & & PHQ-9 & PSS-14 \\
\hline \multirow{7}{*}{$\begin{array}{l}\text { Conforto } \\
\text { em negociar } \\
\text { necessidades } \\
\text { com chefia }\end{array}$} & \multirow{3}{*}{ Sim } & $\mathrm{N}$ & 105,000 & 105,000 \\
\hline & & Média & 8,140 & 26,900 \\
\hline & & D.P. & 6,110 & 8,310 \\
\hline & \multirow{4}{*}{ Não } & $\mathrm{N}$ & 27,000 & 27,000 \\
\hline & & Média & 12,960 & 28,140 \\
\hline & & D.P. & 4,970 & 7,190 \\
\hline & & Sig. & $0,000^{* * *}$ & 0,311 \\
\hline \multirow{7}{*}{$\begin{array}{l}\text { Trabalha em } \\
\text { equipe }\end{array}$} & \multirow{3}{*}{ Sim } & $\mathrm{N}$ & 49,000 & 49,000 \\
\hline & & Média & 11,290 & 29,450 \\
\hline & & D.P. & 5,940 & 6,880 \\
\hline & \multirow{4}{*}{ Não } & $\mathrm{N}$ & 83,000 & 83,000 \\
\hline & & Média & 7,860 & 25,920 \\
\hline & & D.P. & 6,210 & 8,500 \\
\hline & & Sig. & $0,001^{* *}$ & $0,023^{*}$ \\
\hline \multirow{7}{*}{$\begin{array}{l}\text { Recebe ajuda } \\
\text { dos colegas } \\
\text { no trabalho }\end{array}$} & \multirow{3}{*}{ Sim } & $\mathrm{N}$ & 53,000 & 53,000 \\
\hline & & Média & 10,080 & 28,750 \\
\hline & & D.P. & 6,680 & 7,270 \\
\hline & \multirow{4}{*}{ Não } & $\mathrm{N}$ & 79,000 & 79,000 \\
\hline & & Média & 8,490 & 26,200 \\
\hline & & D.P. & 5,800 & 8,510 \\
\hline & & Sig. & 0,319 & 0,114 \\
\hline \multirow{7}{*}{$\begin{array}{l}\text { Recebe apoio } \\
\text { do chefe } \\
\text { em suas } \\
\text { iniciativas }\end{array}$} & \multirow{3}{*}{ Sim } & $\mathrm{N}$ & 39,000 & 39,000 \\
\hline & & Média & 9,180 & 27,240 \\
\hline & & D.P. & 5,900 & 8,240 \\
\hline & \multirow{4}{*}{ Não } & $\mathrm{N}$ & 93,000 & 93,000 \\
\hline & & Média & 9,000 & 27,210 \\
\hline & & D.P. & 6,920 & 7,850 \\
\hline & & Sig. & 0,777 & 0,389 \\
\hline
\end{tabular}

${ }^{*} \mathrm{p}<0,05 ;{ }^{* *} \mathrm{p}<0,01 ;{ }^{* * *} \mathrm{p}<0,001$
Quanto à clareza nas exigências e definição das funções, na comparação entre estresse de servidores que sabem o que o chefe espera deles com aqueles que não sabem, não foi observado valores de significância (Tabela 4). Da mesma forma se portaram os dados para estresse com a variável clareza das deliberações superiores. Com a depressão foi diferente, pois servidores cujos chefes apresentavam deliberações claras e sensatas relataram ser mais deprimidos do que os que sabiam ( $p<0,001$ ). E servidores que não sabiam o que esperar do seu trabalho foram mais acometidos de sintomas depressivos do que os servidores que conheciam as expectativas de seus superiores $(\mathrm{p}<0,01)$.

\section{Tabela 4}

Análise da relação entre percepção de clareza nas exigências e definição das funções dos servidores do INSS de Rondônia e sintomas de estresse percebido e depressão.

\begin{tabular}{|c|c|c|c|c|}
\hline \multicolumn{5}{|c|}{ Clareza nas exigências e definição das funções } \\
\hline & & & PQH-9 & PSS-14 \\
\hline \multirow{7}{*}{$\begin{array}{l}\text { Deliberações } \\
\text { superiores } \\
\text { são claras e } \\
\text { sensatas }\end{array}$} & \multirow{3}{*}{ Sim } & $\mathrm{N}$ & 75,000 & 75,000 \\
\hline & & Média & 7,610 & 26,850 \\
\hline & & D.P. & 6,340 & 8,240 \\
\hline & \multirow{4}{*}{ Não } & $\mathrm{N}$ & 54,000 & 54,000 \\
\hline & & Média & 11,170 & 27,350 \\
\hline & & D.P. & 5,620 & 7,930 \\
\hline & & Sig. & $0,000^{*}$ & 0,750 \\
\hline \multirow{7}{*}{$\begin{array}{l}\text { Sabem o que } \\
\text { esperam do } \\
\text { seu trabalho }\end{array}$} & \multirow{3}{*}{ Sim } & $\mathrm{N}$ & 87,000 & 87,000 \\
\hline & & Média & 7,890 & 26,830 \\
\hline & & D.P. & 6,110 & 8,310 \\
\hline & \multirow{4}{*}{ Não } & $\mathrm{N}$ & 45,000 & 45,000 \\
\hline & & Média & 11,530 & 28,000 \\
\hline & & D.P. & 5,670 & 7,690 \\
\hline & & Sig. & $0,001^{*}$ & 0,705 \\
\hline
\end{tabular}

${ }^{*} \mathrm{p}<0,001$.

\section{Discussão}

A pesquisa não identificou relação entre a assertividade com o estresse percebido e os sintomas depressivos, mas houve relações entre estresse e depressão com as condições de trabalho nos servidores do INSS de Rondônia, o que corrobora com os resultados de outros estudos (Eslami et al., 2016; Menkes, 2012; Pellegrini, Calais, \& Salgado, 2012) e diverge de outras pesquisas que sugerem relação entre a assertividade e estas variáveis (Hirokawa et al., 2012; Ismayilova, Houmod, Alkhasawneh, Shaw \& El-Bassel, 2012; Razayat, 
\& Neyeri, 2014; Segrin, Hanzal, Dornnestein, Taylor \& Domschke, 2007).

Uma possível explicação para esta discrepância pode estar no fato de que a maior parte das pesquisas que validam a correlação da assertividade com estresse e depressão referem-se à amostra de estudantes (Ismayilova et al., 2012; Razayat, \& Neyeri, 2014; Segrin, et al., 2007) ou profissionais que não sejam servidores públicos (Bolsoni-Silva, \& Guerra, 2014; Falcone, Fernandes, \& Sardinha, 2012; Gherardi-Donato et al., 2015; Hirokawa et al., 2012; Wang et al., 2012). É provável que assertividade no ambiente escolar e no contexto do trabalho privado sejam mais eficazes aos usuários agindo de forma a diminuir o estresse e a depressão, quando que no serviço público, ser um elemento que por si só não chega a ser suficiente para dirimir os níveis de estresse a que os trabalhadores estejam submetidos.

Uma outra razão para a divergência entre os dados desta pesquisa e os demais estudos científicos provavelmente esteja no instrumento utilizado, já que não usamos uma escala que tenha como fim específico a mensuração da assertividade e que outros estudos que tenham feito uso de instrumento focado nesta variável tenham encontrado valores mais significativos.

Em se tratando de estresse percebido, depressão e as condições de trabalho, houve significativas relações entre essas variáveis, o que implica em coerência com outros trabalhos científicos (Enns et al., 2015; Santos et al., 2011). Uma das condições de trabalho apontada por esta pesquisa como interveniente na depressão foi a autonomia no contexto laboral, o que representa um alinhamento dessa informação com estudos anteriores que demonstram que os servidores que se sentem livres para gerenciar sua rotina de trabalho e autodeterminar suas atividades são menos propensos a depressão (Gavin, Reisdorfer, Gherardi-Donato, Reis, \& Zanetti, 2015; Enns et al., 2015; Ford, 2012). Os dados indicam que os servidores que se sentiam mais autônomos no trabalho foram quase que duas vezes menos depressivos que aqueles que se percebiam sem autonomia, o que indica a importância que esta condição de trabalho tem para os servidores do INSS/RO.

Durante o estudo observamos que as agências do INSS/RO são altamente controladas. Cada servidor tem agendamentos diários, não podendo ocorrer atrasos, devendo exercer suas funções com pequena autonomia para gerenciar seu tempo e organizar sua forma de trabalho. A insuficiente autonomia percebida pelos servidores parece implicar no desenvolvimento de sentimentos negativos sobre si, descomprometimento, insatisfação e a destituição do significado do seu trabalho, sentimentos típicos da depressão.

A condição de trabalho formada pelo relacionamento interpessoal do trabalhador com a chefia implicou em sintomas depressivos. A pesquisa identificou que os servidores que tinham dificuldades em lidar com seus superiores e sentiam-se desconfortáveis em fazer negociações com a chefia, aqueles que não sabiam o que seus chefes esperam de seus trabalhos, bem como aqueles que percebiam como confusas as deliberações da chefia eram quase duas vezes mais depressivos que os encontrados no grupo inverso. Estes dados despertam preocupação, uma vez que a nota de corte recomendada para identificação de depressão pelo instrumento PHQ-9 é $\geq 10$ (Santos et al., 2013) e, quanto maior a pontuação, pior a gravidade dos indícios depressivos.

Estes dados estão em consonância com a literatura (Monteiro, Oliveira, Ribeiro, Grisa \& Agostini, 2013; Ford, 2012; Santos et al., 2011) e sugerem a hipótese de os estilos de liderança nesta instituição implicarem negativamente nos subordinados, gerando sentimentos que desestimulam as relações entre colaboradores e gestores. É provável também que este estilo de liderança promova uma gestão que limita a autonomia dos servidores, característica já observada no perfil de manifestação da depressão dos funcionários pesquisados.

Outro fator apontado pela pesquisa foi que os servidores que trabalhavam em equipe foram mais estressados que aqueles que executavam seu trabalho de forma individual. Os resultados encontrados foram ligeiramente acima do valor de corte apresentado por Feitosa et al. (2015), mas suficiente para determinar a relativa presença de estresse. Observou-se algo similar para depressão, pois os servidores que trabalhavam em equipe apresentaram média equivalente ao de diagnóstico de depressão moderada (Santos et al., 2013). Estes dados são coerentes com a literatura científica (Santos et al., 2012) e mostram a relação entre trabalhar em equipe, estresse e depressão, provavelmente pela possível interferência de conflitos interpessoais e falta de autonomia dentro da equipe. No INSS o trabalho em equipe é frequente nas agências, onde o número de clientela é bem maior em relação 
àqueles locais em que as atividades são desenvolvidas de forma individual, como a perícia para aposentadoria ou gerência executiva. Desta forma, os servidores das agências lidam constantemente com cobranças das chefias e dos clientes, em contexto laboral onde há falta de clareza sobre como operar funções.

\section{Considerações Finais}

Nesta pesquisa observou-se que a assertividade se relaciona inversamente com estresse e depressão, todavia esta interação não chega a ser significativa a ponto de inibir o estresse e depressão dos servidores. Já algumas condições de trabalho como relação com chefia, autonomia e trabalho em equipe, são significativas principalmente no que concerne à depressão. Nota-se que as relações interpessoais (negociação com superior e trabalho em equipe) estiveram significativamente presentes nos quadros comparativos com os sintomas depressivos e o estresse. $\mathrm{O}$ estudo apresentou limitações em virtude de utilizar como instrumento para verificação da assertividade uma escala que não foi desenvolvida exclusivamente para este fim, o que pode favorecer a fragilidade dos dados referentes à assertividade. Outra limitação diz respeito aos procedimentos utilizados ao fato de serem muitos instrumentos aplicados e terem sido autoadministrados em horário de expediente, o que pode ter contribuído para que os respondentes tenham se cansado e ao chegar no instrumento que verifica a assertividade, tenham respondido com menor atenção e critério. A escassez de estudos na literatura que tenham estudado a interação destas variáveis com servidores públicos também dificultou inferências mais precisas. Sendo assim, há necessidade de outros estudos na área das relações interpessoais e saúde mental de servidores públicos para compreender melhor a dinâmica destas variáveis. Sugere-se a elaboração de políticas institucionais voltadas à saúde mental dos servidores do INSS/RO com valorização de estratégias focadas no aperfeiçoamento dos estilos de lideranças, relações interpessoais positivas, flexibilização do controle no gerenciamento do trabalho e incentivo a atividades complementares à saúde. Atividades da Psicologia como Treinamento de Habilidades Sociais, Treinamento Assertivo, acompanhamento psicológico clínico e em grupo para servidores com consideráveis níveis de estresse e depressão, podem contribuir com o desenvolvimento de relacionamentos interpessoais no trabalho mais saudáveis, bem como minimizar os efeitos do estresse e diminuir as chances do adoecimento de depressão destes servidores públicos. O que por certo tende a beneficiar tantos os servidores como os usuários do sistema previdenciário federal do Brasil.

\section{Referências}

Blanch, J. M. (2003). Condiciones de trabajo. In J. M. Blanch, M. J. Espuny, C. Gala, \& A. Martín (Orgs.), Teoría de las relaciones laborales: Fundamentos (pp. 42-44). Barcelona: UOC.

Bolsoni-Silva, A. T., \& Carrara, K. (2010). Habilidades sociais e análise do comportamento: Compatibilidades e dissensões conceitual-metodológicas. Psicologia em Revista, 16(2), 330-350.

Bolsoni-Silva, A. T., \& Guerra, B. T. (2014). O impacto da depressão para interações sociais de universitários. Estudos e Pesquisas em Psicologia, 14(2), 429-452.

Brasil. Ministério da Previdência Social. (2017). Boletim estatístico da previdência social. Brasília: o autor. Recuperado de: http://www.previdencia.gov.br/wp-content/uploads/2017/04/beps17.02.pdf

Cavalheiro, G. (2010). Sentidos atribuídos ao trabalho por profissionais afastados do ambiente laboral em decorrência de depressão (Dissertação de mestrado). Universidade Federal de Santa Catarina, Florianópolis, SC, Brasil.

Cavalheiro, G., \& Tolfo, S. R. (2011). Trabalho e depressão: Um estudo com profissionais afastados do ambiente laboral. Psico-Usf, 16(2), 241-249. https://doi.org/10.1590/S1413-2712011000200013

Cohen, S., Karmack, T. \& Mermelsteinm, R. (1983). A global measure of perceived stress. J Health Soc Behav. 24(4), 385-96. https://doi.org/10.2307/2136404

Cohen S., \& Williamsom G. M. (1988). Perceived stress in a probability sample of United States. In S. Spacapan, \& S. Oskamp (Orgs.), The social psychology of health: Claremont symposium on applied social psychology. Newbury Park, CA: Sage. 
Constituição da República Federativa do Brasil de 1988. Recuperado de: http://www.senado.gov.br/atividade/ const/con1988/con1988_12.07.2016/CON1988.pdf

Enns, V. C. S., \& Wang, J. (2015). Professional autonomy and work setting as contributing factors to depression and absenteeism in canadian nurses. Nursing Outlook, 63(3), 269-77. https:// doi.org/10.1016/j.outlook.2014.12.014

Eslami, A. A., Rabiei, L., Afzali, S. M., Hamidizadeh, S., \& Masoudi, R. (2016). The effectiveness of assertiveness training of the levels of stress, axiety and depression of high school students. Iranian Red Crescent Medical Journal, 18(1). https://doi.org/10.5812/ircmj.21096

Falcone, M. O., Fernandes, C. S., \& Sardinha, A. (2012). Deficiências em habilidades sociais na depressão: Estudo comparativo. Psicologia: Teoria e Prática, 14(1), 183-196.

Feitosa, F. B. (2014). A depressão pela perspectiva biopsicossocial e a função protetora das habilidades sociais. Psicologia: Ciência e Profissão, 34(2), 488-499.

Feitosa, F. B., Silva, J. F., \& Bezerra, L. A. (2015). Apresentação de uma versão em português da escala de estresse percebido (PSS-14) com índices de precisão. In International Stress Management Association, Anais do congresso da ISMA-BR. Porto Alegre, RS: o autor.

Flynn, M., \& Rudolph, K. D. (2011). Stress generation and adoloescent depression: Contribution of interpesonal stress responses. Journal of Abnormal Child Psychology, 39(8), 1187-1198. https://doi.org/10.1007/s10802-011-9527-1

Ford, M. T. (2012). Job-ocupation misfit na ocupation stressor. Journal of Vocation Behavior, 80(2), 412-421. https:// doi.org/10.1016/j.jvb.2011.10.004

Galvão, A. A. Y. (2015). Previdência social no Rio Grande do Sul: Cenários e interfaces com a saúde do trabalhador (Dissertação de mestrado). Universidade Católica do Rio Grande do Sul, Porto Alegre, RS, Brasil.

Gavin, R. S., Reisdorfer, E., Gherardi-Donato, E. C. S., Reis, L. N., \& Zanetti, A. C. G. (2015). Association between depression, stress, anxiety and alcohol use among civil servants. SMAD: Revista Eletrônica Saúde Mental Álcoole Drogas, 11(1), 2-9. https://doi.org/10.11606/issn.1806-6976.v1lilp2-9

Gherardi-Donato, E. C. S., Cardoso, L., Teixeira, C. A. B., Pereira, S. S., Reisdorfer, E. (2015). Associação entre depressão e estresse laboral em profissionais de enfermagem de nível médio. Revista Latino-Americana de Enfermagem, 23(4), 733-740. https://doi.org/10.1590/0104-1169.0069.2610

Hirokawa, K., Taniguchi, T., Tsuchyia, M., \& Kwakami, N. (2012). Effects of stress management program of hospital staff on their coping strategies and interpessoal behaviors. Industrial Health, 50(6), 487-498. https://doi. org/10.2486/indhealth.MS1358

Ismayilova, L., Houmod, O., Alkhasawneh, E., Shaw, S., \& El-Bassel, N. (2012). Depressive symptoms smong jordanian youth: Results of a national survey. Community Mental Health Journal, 49(1), 133-140. https://doi. org/10.1007/s10597-012-9529-7

Lazarus, R., \& Folkman, S. (1984). Stress, appraisal and coping. New York, NY: Springer.

Menkes, C. (2012). As relações habilidades sociais e estresse ocupacional em submarinistas da Marinha do Brasil (Dissertação de mestrado). Universidade do Estado do Rio de Janeiro, Rio de Janeiro, RJ, Brasil.

Monteiro, J. K., Oliveira, A. L. L., Spara, C., Grisa, G. H., \& Agostini, N. (2013). Mental illness of workers in intensive care units. Psicologia: Ciência e Profissão, 33(2), 366-379. https:// doi.org/10.1590/S1414-98932013000200009

Motta, C. C. L. (2011). Depressão: Sua compreensão e significados à luz da prática dos psicólogos no contexto de uma rede municipal de saúde mental (Tese de doutorado). Universidade Federal de Santa Catarina. Florianópolis, SC.

Hackbarth, A. A. H., Neto, \& Stein, C. E. (2003). Uma abordagem dos testes não-paramétricos com utilização do Excel. Recuperado de: http://home.furb.br/efrain/matematica/minicurso/artigo_11_09_2003.doc.

Nunes, C. H. S. S. (2007). Lançamento da escala fatorial de extroversão (EFEx) e escala fatorial de socialização (EFS). Avaliação Psicológica, 6(1), 103-106.

Pellegrini, C. F. S., Calais, S. L., \& Salgado, M. H. (2012). Habilidades sociais e administração de tempo no manejo do estresse. Arquivos Brasileiros de Psicologia, 64(3), 110-129.

Rezayat, F., \& Nayeri, N. D. (2014). The level of depression and assertivenes among nursing students. International Journal of Community Based Nursing and Midwifery, 2(3), 177-184. 
Santos, I. S., Tavares, B. F., Munhoz, T. N., Almeida, L. S. P., Silva, N. T. B., Tams, B. D. et al. (2013). Sensibilidade e especificidade do Patient Health Questionnaire-9 (PHQ- 9) entre adultos da população geral. Caderno de Saúde Pública, 29(8), 1533-1543. https://doi.org/10.1590/0102-311X00144612

Santos, C. L. M., Rodrigues, C. L. P., Silva, L. B. S., Bakke, H. A., Leite, A. S. M., \& Leal, M. M. A. (2011). Fatores de estresse na atividade de médicos em João Pessoa (PB, Brasil). Production, 21(1), 181-189. https:// doi.org/10.1590/ S0103-65132011005000003

Sarkova, M., Sarkova, M., Bacikova-Sleskova, M., Orosova, O., Geckova, A. M., Katreniakova, Z. et al. (2013). Associations between assertiveness, psychological well-being, and self-esteem in adolescents. Journal of Applied Social Psychology, 43(1), 147-154. https://doi.org/10.1111/j.1559-1816.2012.00988.x

Segrin, C., Hanzal. A., Dornnestein, C., Taylor, M., \& Domschke, T. J. (2007). Social skills, psychological wellbeing, and the mediating role of perceived stress. Anxiety, Stress, \& Coping, 20(3), 321-329. https://doi. org/10.1080/10615800701282252

Uliaszek, A. A., Zinbarg, R. E., Mineka, A., Craske, M. G., Sutton, J. M., Griffith, J.W. et al. (2010). The role of neuroticism and extraversion in the stress-anxiety and stress-depression relationships. Anxiety, Stress, and Coping, 23(4), 365-381. https://doi.org/10.1080/10615800903377264

\section{Leandro Aparecido Fonseca Missiatto}

Mestre em Psicologia pela Faculdade de Ciências Biomédicas de Cacoal. Cacoal - RO. Brasil.

E-mail: leandro_afonseca@hotmail.com

$$
\text { (iD) https://orcid.org/0000-0002-6532-735X }
$$

\section{Fabio Biasotto Feitosa}

Pós-Doutor em Psicologia pela Universidade Federal de Rondônia, Porto Velho - RO. Brasil.

E-mail:fabiobfeitosa@gmail.com

(iD https://orcid.org/0000-0001-6440-4993

\section{Tomás Daniel Menéndez Rodríguez}

Pós-Doutor em Matemática pela Universidade Federal de Rondônia, Porto Velho - RO. Brasil.

E-mail:tomasdanielm@gmail.com

(iD) https:// orcid.org/0000-0002-4425-1261

Endereço para envio de correspondência:

SEDE: Av Cuiabá, 3087 - Jd. Clodoaldo. CEP: 76963-665. Cacoal - RO. Brasil.

Recebido 17/11/2017

Reformulado 27/07/2018

Aceito 17/09/2018

Received $11 / 17 / 2017$

Reformulated 07/27/2018

Approved 09/17/2018

Recibido 17/11/2017

Reformulado 27/07/2018

Aceptado 17/09/2018 
Como citar: Missiatto, L. A. F., Feitosa, F. B., \& Rodríguez, T. D. M. (2019). Correlações entre assertividade, condições laborais, estresse e depressão de servidores do INSS/RO. Psicologia: Ciência e Profissão, 39, 1-12. https://doi.org/10.1590/1982-3703003188004

How to cite: Missiatto, L. A. F., Feitosa, F. B., \& Rodríguez, T. D. M. (2019). Correlations between assertiveness, labor conditions, stress and depression of government employees INSS/RO. Psicologia: Ciência e Profissão, 39, 1-12. https://doi.org/10.1590/1982-3703003188004

Cómo citar: Missiatto, L. A. F., Feitosa, F. B., \& Rodríguez, T. D. M. (2019). Correlaciones entre Asertividad, Condiciones laborales, estrés y depresión de servidores del INSS/RO. Psicologia: Ciência e Profissão, 39, 1-12. https://doi.org/10.1590/1982-3703003188004 